A pinhole gamma camera with optical depth-of-interaction elimination

This article has been downloaded from IOPscience. Please scroll down to see the full text article. 2009 Phys. Med. Biol. 54 N267

(http://iopscience.iop.org/0031-9155/54/13/N02)

View the table of contents for this issue, or go to the journal homepage for more

Download details:

IP Address: 131.180.130.114

The article was downloaded on 21/12/2010 at 09:45

Please note that terms and conditions apply. 
NOTE

\title{
A pinhole gamma camera with optical depth-of-interaction elimination
}

\author{
Marc A N Korevaar ${ }^{1,2}$, Jan W T Heemskerk ${ }^{1,2}$ and Freek J Beekman ${ }^{1,2,3}$ \\ ${ }^{1}$ Department of Nuclear Medicine, Image Sciences Institute, University Medical Center Utrecht, \\ Heidelberglaan 100, 3584 CG, Utrecht, The Netherlands \\ ${ }^{2}$ Section of Radiation Detection and Medical Imaging, Department of R3, Applied Sciences, \\ Delft University of Technology, Mekelweg 15, 2629 JB, Delft, The Netherlands \\ ${ }^{3}$ Molecular Imaging Labs (MILABS), Universiteitsweg 100, 3584 CG, Utrecht, The Netherlands \\ E-mail: f.j.beekman@tudelft.nl
}

Received 21 November 2008, in final form 15 May 2009

Published 12 June 2009

Online at stacks.iop.org/PMB/54/N267

\begin{abstract}
The performance of pinhole single photon emission computed tomography (SPECT) depends on the spatial resolution of the gamma-ray detectors used. Pinhole cameras suffer from strong resolution loss due to the varying depth-ofinteraction (DOI) of gamma quanta that enter the detector material at an angle. We eliminate DOI effects in a scintillation gamma camera via a dedicated optic fiber bundle that acts as a focusing collimator for light generated in a scintillation crystal. A curved crystal is connected to a concavely shaped fiberoptic bundle such that the fibers connect perpendicularly to the crystal's convex surface and point straight at the pinhole opening. Limiting the fiber numerical apertures can be used to suppress resolution losses due to light spread. Here we demonstrate experimentally that this prototype position-sensitive gamma sensor successfully eliminates DOI effects, and has an intrinsic resolution of better than $280 \mu \mathrm{m}$ full width at half maximum with an interaction probability of $67 \%$ for $140 \mathrm{keV}$ photons. Therefore, the detector has great potential for increasing the resolution of pinhole SPECT.
\end{abstract}

(Some figures in this article are in colour only in the electronic version)

\section{Introduction}

Today, the imaging of radio-labeled tracers with gamma cameras, whether in planar or single photon emission computed tomography (SPECT) mode, forms the most frequently used clinical molecular imaging procedure. SPECT imaging of small animals has rapidly gained popularity as a key tool for the development of tracers, development of methods and (radio-labeled) agents for cancer therapy and to study animal models of human disease (Jaszczak et al 1994, McElroy et al 2002, Liu et al 2002, Schramm et al 2003, Furenlid 
et al 2004, Beekman et al 2005, Meikle et al 2005, Metzler et al 2005, Vastenhouw et al 2007, Vastenhouw and Beekman 2007, Beekman and van der Have 2007). A key advantage of SPECT over optical molecular imaging is that the tracers can rapidly be translated to clinical application because gamma radiation has much lower attenuation and scatter in tissue than optical photons. Instrumental in these developments is the recent introduction of sub$\mathrm{mm}$ and sub-half-mm resolution SPECT systems with high quantitative accuracy, mostly based on pinhole collimation technology. Pinholes cameras also have high potential for improving clinical SPECT devices. However, limited resolution and bulkiness of gammaray detectors can be perceived as the largest bottleneck to further improvement of SPECT device performance (Barber et al 1993, Rogulski et al 1993, Beekman and Vastenhouw 2004, Rentmeester et al 2007).

Commonly used scintillation detectors have an intrinsic resolution of a couple of millimeters. In these detectors, the interaction position and energy of gamma quanta are extracted from the scintillation flashes via an array of photomultiplier tubes (PMTs). To improve detection accuracy, a vast amount of research is directed at finding reliable alternatives to PMTs. In one class of novel detectors, these PMTs are replaced by low-noise CCDs with fast read-out capabilities. In order to benefit from the high-resolution light detection capabilities of CCDs novel structured scintillation crystals are being developed.

Recently investigated methods to obtain a much higher spatial resolution include sensors with scintillation crystals consisting of parallel bundles of tiny CsI needles ('columnar crystals') that are optically connected to CCDs. The CCDs are suitable for operating at high frame rates (e.g. $50 \mathrm{~Hz}$ ) (Nagarkar et al 1996, Miyata et al 2004, de Vree et al 2005, Teo et al 2006, Heemskerk et al 2007, Meng and Fu 2008), which enables one to obtain images in which scintillation light flashes have a very high likelihood to be spatially separated; thus, it is possible to detect the gamma photons individually (photon counting). The use of crystals consisting of columns with reflecting sides suppresses spatial resolution losses due to light spreading. At present, however, the gamma-ray interaction probability is low (typically $<30 \%$ at $140 \mathrm{keV}$ ) due to the limited length of needles that can be manufactured. Moreover, when photons enter the crystal surface at an angle, varying depth-of-interaction (DOI) significantly reduces spatial resolution. The goal of the present work is to improve the interaction probability of a CCD-based detector while simultaneously eliminating the associated resolution degrading the DOI effects of pinhole SPECT.

\section{Methods}

The principle of the camera with optical cone-beam collimation (OCC) is shown in figure 1 . Parallax errors caused by gamma-rays entering the crystal at an angle (the DOI effect) and light spreading effects are eliminated using a $3 \mathrm{~mm}$ thick curved monolithic $\mathrm{CsI}(\mathrm{Tl})$ crystal which, together with a dedicated fiber-optic bundle, acts as a focusing collimator (OCC) (Beekman 2007a, 2007b, 2008). The $3 \mathrm{~mm}$ thick curved monolithic crystal with a diameter of $20 \mathrm{~mm}$ was machined from a larger crystal block. The dedicated fiber-optic bundle was machined from a 1:1.67 magnifying taper. The magnifying taper consists of many glass fibers, of $6 \mu \mathrm{m}$ diameter, that propagate the light from one end to the other by means of total internal reflection (Burle 2000). The OCC is optically coupled to an electron-multiplying CCD (EMCCD) using Bicron BC-630 silicone optical grease. Fibers with a limited numerical aperture (N.A.) suppress detection of photons whose angle of incidence is too large.

The fibers are directed at the pinhole, which is also at the focal point of the convex side of the crystal. The fibers are normal to the crystal surface. The crystal and fiber-optics bundle are shown in figure 2. The pinhole diameter was $100 \mu \mathrm{m}$, the point source diameter was 


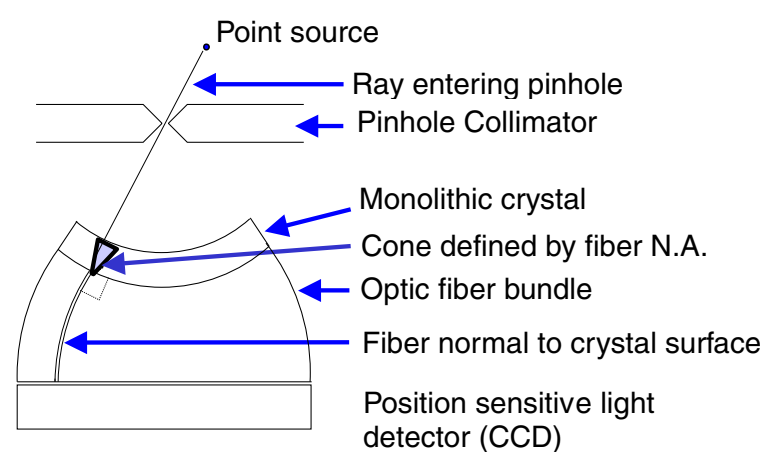

Figure 1. Principle of depth-of-interaction elimination through optical cone-beam collimators (OCC). Gamma rays enter the crystal at an angle of $30^{\circ}$.

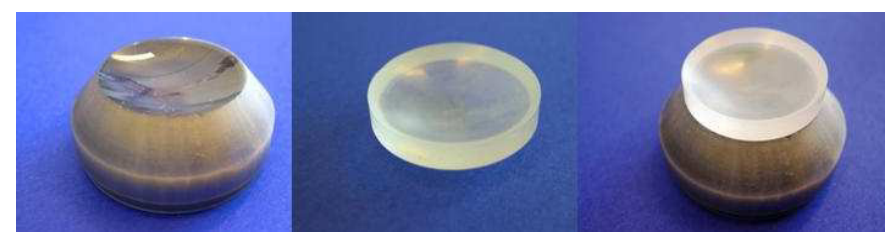

Figure 2. Left: optical cone-beam collimator (OCC); center: curved crystal; right: OCC and crystal stacked.

$300 \mu \mathrm{m}$, the source activity was $100 \mathrm{MBq}$, the measurement time was 4 min and the count rate was approx. 50 counts $\mathrm{s}^{-1}$. The pinhole-to-source distance was $20 \mathrm{~mm}$, the pinholeto-crystal distance was $21 \mathrm{~mm}$ and the opening angle $\alpha$ of the gold pinhole was $62^{\circ}$. To calculate the full width at half maximum (FWHM) of the radiation spot at the crystal surface, the non-Gaussian pinhole response and point source shape were taken into account using the convolution method described in Barrett and Swindell (1981). The pinhole transmission was calculated using the method described in Accorsi and Metzler (2004). Assuming a uniform and spherical point source, this results in a radiation spot on the detector of $274 \mu \mathrm{m}$ FWHM for the point source directly above the pinhole and $229 \mu \mathrm{m}$ FWHM for the point source at an angle of $30^{\circ}$. The intrinsic spatial resolution was determined by correcting the measured resolution for the radiation spot.

The OCC is compared with the optical parallel collimator (OPC) which consists of a flat CsI(Tl) scintillator of the same thickness $(3 \mathrm{~mm})$ on top of a straight fiber optic taper.

We used an EMCCD (type CCD97 from E2V Technologies). This CCD has an internal gain to reduce sensitivity to read-out noise, even at high read-out speeds (e.g. several megapixels $\mathrm{s}^{-1}$ ). The internal gain is achieved by electron multiplication (avalanche multiplication or impact ionization) in the gain register. To reduce the dark current, the EMCCD was cooled to $-50{ }^{\circ} \mathrm{C}$, using a Peltier element. The CCD was operated in photoncounting mode (Beekman and de Vree 2005), in which the individual frames are analyzed by a scintillation detection algorithm (Korevaar et al 2009) to detect the individual gamma quanta. In order to cover the whole field of view, the CCD was moved relative to the OCC in between measurements, but with the use of our future larger CCDs, this will no longer be necessary. 


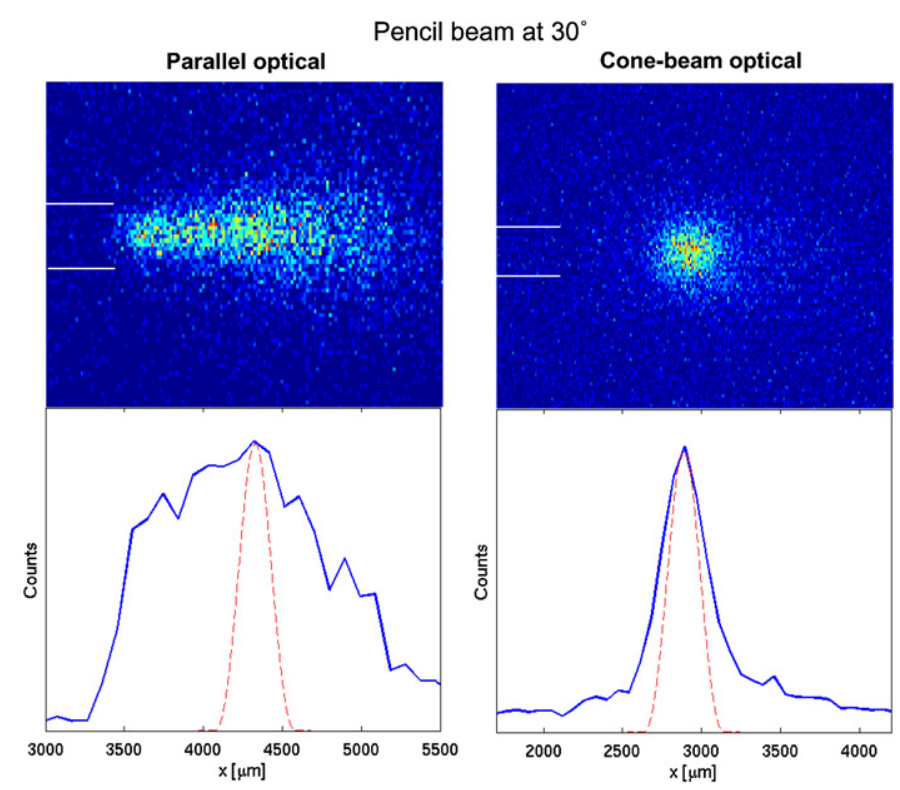

Figure 3. Left: parallel beam collimator (OPC); right: optical cone-beam collimator (OCC). The OCC strongly suppresses depth-of-interaction-induced image degradation. The solid (blue) line is the measured profile and the dashed (red) line represents the spot on the detector when no detector blurring would be present (only blurring due to source and pinhole size). The $x$-axis of both figures has been scaled to the $\mathrm{CsI}(\mathrm{Tl})$ crystal (i.e. multiplied by the demagnification factor of the taper).

Table 1. Spatial resolution measurements for OCC and OPC.

\begin{tabular}{llll}
\hline Angle & $\begin{array}{l}\text { Type of optical } \\
\text { collimation }\end{array}$ & $\begin{array}{l}\text { FWHM } \\
\text { measured }\end{array}$ & $\begin{array}{l}\text { FWHM intrinsic } \\
\text { (corrected for source } \\
\text { size and pinhole size) }\end{array}$ \\
\hline $30^{\circ}$ & Parallel-beam & $1460 \mu \mathrm{m}$ & $1442 \mu \mathrm{m}$ \\
$30^{\circ}$ & Cone-beam & $360 \mu \mathrm{m}$ & $278 \mu \mathrm{m}$ \\
$0^{\circ}$ & Parallel-beam & $386 \mu \mathrm{m}$ & $272 \mu \mathrm{m}$ \\
$0^{\circ}$ & Cone-beam & $389 \mu \mathrm{m}$ & $276 \mu \mathrm{m}$ \\
\hline
\end{tabular}

\section{Results}

Initial results indicate that for the pre-eminent isotope in SPECT (Tc-99m, $141 \mathrm{keV}$ ), pinhole imaging can be performed with an intrinsic resolution of approx. $278 \mu \mathrm{m}$ (FWMH, corrected for the projected spot), an interaction probability of $67 \%$ and an energy resolution of $89 \%$ at $141 \mathrm{keV}$. Figure 3 shows the isolated effects of using the curved crystal with OCC, comparing it with a flat crystal CsI crystal (also $3 \mathrm{~mm}$ thick) combined with an OPC. It shows that the effects of DOI are adequately suppressed with OCC.

Table 1 shows the measured and intrinsic resolution (corrected for the projected spot size) of OPC versus OCC for the multi-scale algorithm (Korevaar et al 2009) that takes into account the varying light spread as a function of depth. As could be expected for gamma rays incident at a normal angle, the differences between OPC and OCC are very small. 


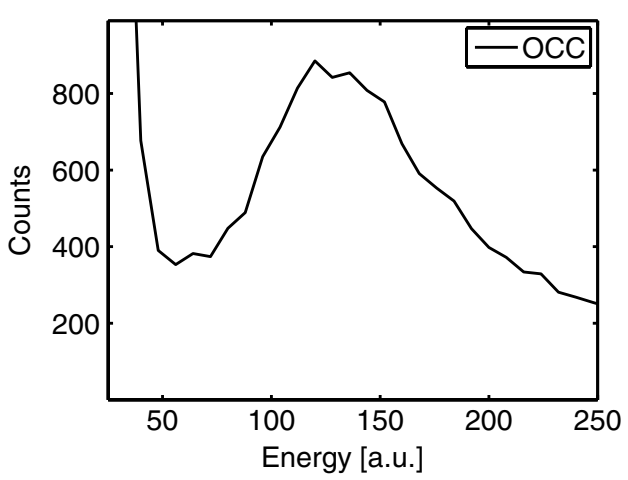

Figure 4. Energy spectrum of Tc-99m measured with the optical cone-beam collimator (OCC).

Figure 4 shows the energy spectrum of the OCC-based detector for a Tc-99m source with an $89 \%$ energy resolution at $141 \mathrm{keV}$.

\section{Discussion and conclusions}

This paper presents the first pinhole gamma camera with intrinsic elimination of spatial resolution losses due to varying DOI. In the first prototype, the intrinsic resolution of the camera is already below $280 \mu \mathrm{m}$. The energy and spatial resolution of future OCC-based detectors may be further improved by e.g. (i) using advanced statistical methods to characterize and localize scintillation events, (ii) fabricating crystals with higher density, better spectral matching or higher light yield, (iii) using light sensors with improved noise characteristics and (iv) using new larger CCDs, currently under development for our group. Such larger CCDs will enable us to perform imaging using the full field of view. A point of further research is the effect of the numerical aperture of the optical fibers on the detector performance.

\section{Acknowledgments}

This work was sponsored in part by The Netherlands Organization for Scientific Research (NWO), grant 917.36.335 and by the Department of Economic Affairs IOP photonics grant IPD067766. We thank Frans van der Have, Marina Gaikhorst and Rene Nouse for support in production, radiation safety and transport of Tc-99m point sources.

\section{References}

Accorsi R and Metzler S D 2004 Analytic determination of the resolution-equivalent effective diameter of a pinhole collimator IEEE Trans. Med. Imaging 23 750-63

Barber H B, Barrett H H, Dereniak E L, Hartsough N E, Perry D L, Roberts P C T, Rogulski M M, Woolfenden J $\mathrm{M}$ and Young E T 1993 A gamma-ray imager with multiplexer read-out for use in ultra-high-resolution brain SPECT IEEE Trans. Nucl. Sci. 40 1140-4

Barrett H H and Swindell W 1981 Radiological Imaging: The Theory of Image Formation, Detection, and Processing (New York: Academic) pp 121-3

Beekman F J and de Vree G A 2005 Photon-counting versus an integrating CCD-based gamma camera: important consequences for spatial resolution Phys. Med. Biol. 50 N109-19

Beekman F J and Van Der Have F 2007 The pinhole: gateway to ultra-high resolution three-dimensional radio-nuclide imaging Eur. J. Nucl. Med. Mol. Imaging 34 151-61 
Beekman F J and Vastenhouw B 2004 Design and simulation of a high-resolution stationary SPECT system for small animals Phys. Med. Biol. 49 4579-92

Beekman F J 2007a Radiation detection apparatus Patent application WO 2007/011214 A1

Beekman F J 2007b Scintillation camera for producing image, has scintillation material on side facing away from detector provided with antireflective layer transparent to high-energy radiation, and light guides are located in front of detector Patent application NL1029558 (C1)

Beekman F J 2008 Radiation detection apparatus Patent application EP1915640 (A1)

Beekman F J, Van Der Have F, Vastenhouw B, Van Der Linden A J A, van Rijk P P, Burbach J P H and Smidt M P 2005 U-SPECT-I: a novel system for submillimeter-resolution tomography with radiolabeled molecules in mice J. Nucl. Med. 46 1194-200

Burle 2000 Fiber optics: theory and application Technical Memorandum http://www.burle.com/cgi-bin/ byteserver.pl/pdf/tp217.pdf

de Vree G A, Westra A H, Moody I, van der Have F, Ligtvoet K M and Beekman F J 2005 Photon-counting gamma camera based on an electron-multiplying CCD IEEE Trans. Nucl. Sci. 52 580-8

Furenlid L R, Wilson D W, Chen Y C, Kim H, Pietraski P J, Crawford M J and Barrett H H 2004 FastSPECT II: a second-generation high-resolution dynamic SPECT imager IEEE Trans. Nucl. Sci. 51 631-5

Heemskerk J W T, Westra A H, Linotte P M, Ligtvoet K M, Zbijewski W and Beekman F J 2007 Front-illuminated versus back-illuminated photon-counting CCD-based gamma camera: important consequences for spatial resolution and energy resolution Phys. Med. Biol. 52 N149-62

Jaszczak R J, Li J Y, Wang H L, Zalutsky M R and Coleman R E 1994 Pinhole collimation for ultra-high-resolution, small-field-of-view spect Phys. Med. Biol. 39 425-37

Korevaar M A N, Heemskerk J W T, Goorden M C and Beekman F J 2009 Multi-scale algorithm for improved scintillation detection in a CCD-based gamma camera Phys. Med. Biol. 54 831-42

Liu Z L, Kastis G A, Stevenson G D, Barrett H H, Furenlid L R, Kupinski M A, Patton D D and Wilson D W 2002 Quantitative analysis of acute myocardial infarct in rat hearts with ischemia-reperfusion using a high-resolution stationary SPECT system J. Nucl. Med. 43 933-9

McElroy D P, MacDonald L R, Beekman F J, Wang Y C, Patt B E, Iwanczyk J S, Tsui B M W and Hoffman E J 2002 Performance evaluation of a-SPECT: a high resolution desktop pinhole SPECT system for imaging small animals IEEE Trans. Nucl. Sci. 49 2139-47

Meikle S R, Kench P, Kassiou M and Banati R B 2005 Small animal SPECT and its place in the matrix of molecular imaging technologies Phys. Med. Biol. 50 R45-61

Meng L J and Fu G 2008 Investigation of the intrinsic spatial resolution of an intensified EMCCD scintillation camera IEEE Trans. Nucl. Sci. 55 2508-17

Metzler S D, Jaszczak R J, Patil N H, Vemulapalli S, Akabani G and Chin B B 2005 Molecular imaging of small animals with a triple-head SPECT system using pinhole collimation IEEE Trans. Med. Imaging 24 853-62

Miyata E, Miki M, Tawa N, Kamiyama D and Miyaguchi K 2004 Development of new X-ray imaging device sensitive to $0.1-100 \mathrm{keV}$ Nucl. Instrum. Methods A $\mathbf{5 2 5} 122-5$

Nagarkar V V, Gordon J S, Vasile S, Gothoskar P and Hopkins F 1996 High resolution X-ray sensor for non destructive evaluation IEEE Trans. Nucl. Sci. 43 1559-63

Rentmeester M C M, Van Der Have F and Beekman F J 2007 Optimizing multi-pinhole SPECT geometries using an analytical model Phys. Med. Biol. 52 2567-81

Rogulski M M, Barber H B, Barrett H H, Shoemaker R L and Woolfenden J M 1993 Ultra-high-resolution brain spect imaging-simulation results IEEE Trans. Nucl. Sci. $401123-9$

Schramm N U, Ebel G, Engeland U, Schurrat T, Behe M and Behr T M 2003 High-resolution SPECT using multipinhole collimation IEEE Trans. Nucl. Sci. 50 315-20

Teo B K, Shestakova I, Sun M, Barber W C, Nagarkar V V and Hasegawa B H 2006 Evaluation of a EMCCD detector for emission-transmission computed tomography IEEE Trans. Nucl. Sci. 53 2495-9

Vastenhouw B and Beekman F 2007 Submillimeter total-body murine imaging with U-SPECT-I J. Nucl. Med. 48 487-93

Vastenhouw B, Van Der Have F, Van Der Linden A J A, von Oerthel L, Booij J, Burbach J P H, Smidt M P and Beekman F J 2007 Movies of dopamine transporter occupancy with ultra-high resolution focusing pinhole SPECT Mol. Psychiatr. 12 984-7 